\title{
Primary Chemical Reactions Induced by Transformation of Radioactive Nuclei in Solids at Low Temperatures. Investigation by Means of the Emission Mössbauer and Positron Spectroscopies
}

\author{
V.M. Byakov ${ }^{a}$, L.A. Kulikov ${ }^{b}$, Y.D. Perfil'EV ${ }^{b}$ \\ AND S.V. STEPANOV ${ }^{a}$ \\ ${ }^{a}$ Institute for Theoretical and Experimental Physics, 117218, Moscow, Russia \\ ${ }^{b}$ Chemical Department, Lomonosov Moscow State University \\ Leninskie Gory, 119899, Moscow, Russia
}

\begin{abstract}
A set of early chemical reactions in grapes of ionization (blobs) arising in tracks of fast electrons and positrons in liquids as well as formed by the Auger electrons in frozen aqueous media around decaying Mössbauer ${ }^{57} \mathrm{Co}$ or ${ }^{119 \mathrm{~m}} \mathrm{Sn}$ nuclei is suggested. The mechanism predicts a correlated variation of the formation probabilities of intrablob final products, namely $\mathrm{Fe}^{2+}-\mathrm{Fe}^{3+}$ or $\mathrm{Sn}^{2+}-\mathrm{Sn}^{4+}$ ions, positronium atom and molecular hydrogen with variation of temperature, degree of crystallinity, concentration of electron scavengers. These correlations indicate on similarity of chemical processes as in blobs created by ${ }^{57} \mathrm{Co}$ and ${ }^{119 \mathrm{~m}} \mathrm{Sn}$ after their decay as well as in blobs produced in tracks of fast positrons and electrons.
\end{abstract}

PACS numbers: 61.80.Fe, 36.10.Dr, 78.70.Bj

\section{Introduction}

We present a unified interpretation of chemical reactions in molecular solids, induced by fast $\mathrm{e}^{+}$and $\mathrm{e}^{-}$irradiation as well as by radioactive nuclear decay accompanied by emission of the Auger electrons and subsequent ionizations. Investigations of these processes are obviously interesting in view of understanding of physico-chemical transformations, taking place in media, containing radioactive 
isotopes. It is also important for correct treatment of the data obtained by the Mössbauer spectroscopy, radiation chemistry and $\mathrm{e}^{+}$and Ps chemistry. Primarily we shall consider reactions in frozen aqueous solutions $(77 \mathrm{~K})$, nevertheless, our findings shed light on intratrack processes at normal conditions.

If an emission Mössbauer spectroscopy (EMS) is applied, a small amount of special ("Mössbauer") isotopes $\left({ }^{57} \mathrm{Co},{ }^{119 \mathrm{~m}} \mathrm{Sn}\right.$ ) are added into investigated solid state system. A common property of these isotopes consists in production of the Auger ionization of the "daughter" atoms $\left({ }^{57} \mathrm{Fe},{ }^{119} \mathrm{Sn}\right)$, caused by E-capture or converted isomeric transition. These processes precede emission of the Mössbauer $\gamma$-quantum by ${ }^{57} \mathrm{Fe}$ or ${ }^{119} \mathrm{Sn}$, which contains physico-chemical information about the daughter atoms and their environment [1].

Auger ionization converts the daughter atom into multi-charge ion (electric charge of ${ }^{57} \mathrm{Fe}$ ion could reach +8 ). This process is accompanied by emission of a large number of the Auger electrons with energies mainly above $10^{2} \mathrm{eV}$. Together with X-rays within $10^{-14} \mathrm{~s}$ they produce ionization of many molecules in a spherical environment (of a nanometer scale) of the ion. In the literature on radiation chemistry such a cloud of tens or hundreds ion-electron pairs is called as blob $[2,3]$. Here we shall call it as the Auger blob.

The blob is a structural element of the track of any ionizing particle. Usually it consists of 30-50 ion-electron pairs. It is created by a secondary electron with the energy $10^{2}-10^{3} \mathrm{eV}$. A terminal part of the positron track is also the blob [3].

\section{Chemical processes in blobs}

Within $\approx 10^{-7} \mathrm{~s}$ after its birth ${ }^{57} \mathrm{Fe}$ nucleus emits a Mössbauer $\gamma$-quantum $14.4 \mathrm{keV}$, which is registered by a detector. It is important that the energy of the photon depends on the number of electrons, composing electronic shell of ${ }^{57} \mathrm{Fe}^{n+}$ ion, and, therefore, conveys information about $n$, at a time when it was emitted [1]. Initially electron affinity of ${ }^{57} \mathrm{Fe}^{n+}$ significantly exceeds first ionization potential of the surrounding molecules and neutralization of ${ }^{57} \mathrm{Fe}^{n+}$ proceeds by picking up electrons from them. By the time moment of emission of the Mössbauer photon the ion usually restores its electron shell up to one of its chemically stable states $\left(\mathrm{Fe}^{3+}\right.$ or $\left.\mathrm{Fe}^{2+}\right)$, mainly $\mathrm{Fe}^{3+}$. We assume that reduction of $\mathrm{Fe}^{3+}$ to $\mathrm{Fe}^{2+}$ proceeds via recombination with quasi-free electrons, $\mathrm{e}^{-}$, of the Auger blob

$$
\mathrm{Fe}^{3+}+\mathrm{e}^{-} \rightarrow \mathrm{Fe}^{2+} .
$$

Reaction (1) is similar to the Ps formation in the positron blob

$$
\mathrm{e}^{+}+\mathrm{e}^{-} \rightarrow \text { Ps. }
$$

Therefore one may expect that in a given medium the formation probabilities of $\mathrm{Fe}^{2+}$ and Ps must be proportional to each other. Moreover, radiolytic hydrogen $\left(\mathrm{H}_{2}\right)$ in water is also a product of intrablob reaction (6) [3]. If so, the yields of $\mathrm{Fe}^{2+}$, Ps and $\mathrm{H}_{2}$ have to correlate in aqueous solutions. Reactions (1)-(2) have 
to be supplemented with reactions, taking place in blobs of fast electrons, with participation of the radiolytic products of the medium. In frozen aqueous solutions the most important reactions are the following $[2,3]$ :

ionization of water molecules by the Auger electrons $\left(\mathrm{e}^{-* *}\right)$ :

$\mathrm{e}^{-* *}+\mathrm{H}_{2} \mathrm{O} \rightarrow \mathrm{e}^{-*}+\mathrm{H}_{2} \mathrm{O}^{+}+\mathrm{e}^{-*}$

thermalization of "hot" electrons $\left(\mathrm{e}^{-*}\right)$ :

$\mathrm{e}^{-*} \rightarrow \mathrm{e}^{-}+\mathrm{H}_{2} \mathrm{O}^{*}$

trapping of thermalized electrons $\left(\mathrm{e}^{-}\right)$on structural traps with formation of trapped electrons $\left(\mathrm{e}_{\mathrm{tr}}^{-}\right)$:

$\mathrm{e}^{-}+\operatorname{trap} \rightarrow \mathrm{e}_{\mathrm{tr}}^{-}$,

other chemical reactions:

$\mathrm{e}^{-}+\left(\mathrm{H}_{2} \mathrm{O}, \mathrm{H}_{2} \mathrm{O}\right)^{+\cdot} \rightarrow \mathrm{H}_{2}+2 \dot{\mathrm{O}} \mathrm{H}$

$\mathrm{H}_{2} \mathrm{O}^{+\cdot}+\mathrm{H}_{2} \mathrm{O} \rightarrow \mathrm{H}_{3} \mathrm{O}^{+}+\dot{\mathrm{O}} \mathrm{H}$

$\mathrm{e}^{-}+\dot{\mathrm{OH}} \rightarrow \mathrm{OH}^{-}$

$\mathrm{H}_{3} \mathrm{O}^{+}+\mathrm{OH}^{-} \rightarrow 2 \mathrm{H}_{2} \mathrm{O}$

$\mathrm{e}^{-}+\mathrm{H}_{3} \mathrm{O}^{+} \rightarrow \dot{\mathrm{H}}+\mathrm{H}_{2} \mathrm{O}$

$\mathrm{e}^{-}+\mathrm{S} \rightarrow \mathrm{S}^{-}$

$\mathrm{H}_{2} \mathrm{O}^{+.}+\mathrm{S} \rightarrow\left\{\begin{array}{l}\dot{\mathrm{O}} \mathrm{H}+\mathrm{SH}^{+} \\ \mathrm{H}_{2} \mathrm{O}+\mathrm{S}^{+} .\end{array}\right.$

\section{Quantitative consequences of the model}

From these reactions one may obtain quantitative relations concerning variation of the yields of ortho-Ps, $I_{o-\mathrm{Ps}}$, radiolytic hydrogen, $G_{\mathrm{H} 2}$, doubly charged Fe ion, $R\left(\mathrm{Fe}^{2+}\right)$, and hydrated electron, $G\left(\mathrm{e}_{\mathrm{aq}}^{-}\right)$vs. concentration $c_{\mathrm{S}}$ of electron scavenger $(\mathrm{S})$. Inhibition of these yields may be approximated by the following expressions $[3-5]$ :

$$
\begin{aligned}
& I_{o-\mathrm{Ps}}=I_{o-\mathrm{Ps}}^{0} /\left(1+p c_{\mathrm{S}}\right), \\
& G_{\mathrm{H} 2}=G_{\mathrm{H} 2}^{0} /\left(1+h c_{\mathrm{S}}\right), \\
& R\left(\mathrm{Fe}^{2+}\right)=R^{0}\left(\mathrm{Fe}^{2+}\right) \exp \left(-f c_{\mathrm{S}}\right), \\
& G\left(\mathrm{e}_{\mathrm{aq}}^{-}\right)=G^{0}\left(\mathrm{e}_{\mathrm{aq}}^{-}\right) \exp \left(-q c_{\mathrm{S}}\right) .
\end{aligned}
$$

Inhibition coefficients of $o-\mathrm{Ps}, \mathrm{H}_{2}, \mathrm{Fe}^{2+}$ and $\mathrm{e}_{\mathrm{aq}}^{-}\left(\mathrm{e}_{\mathrm{tr}}^{-}\right)$, namely $p, h, f$, and $q$ characterize the reaction ability of $\mathrm{S}$ towards thermalized quasi-free electrons $\left(\mathrm{e}^{-}\right)$, 
which we consider as the $\mathrm{H}_{2}, o-\mathrm{Ps}, \mathrm{Fe}^{2+}$ and $\mathrm{e}_{\mathrm{aq}}^{-}\left(\mathrm{e}_{\mathrm{tr}}^{-}\right)$precursors. Each coefficient is proportional to the rate constant $k\left(\mathrm{e}^{-}+\mathrm{S}\right)$ of quasi-free electron with $\mathrm{S}$ :

$$
p \propto h \propto f \propto q \propto k\left(\mathrm{e}^{-}+\mathrm{S}\right) .
$$

Below we shall present experimental data, which prove consequences (13)-(16) of the model. Unfortunately, we have no possibility to compare data from different experiments in identical conditions. There are few radiation-chemical and positron annihilation data, obtained in frozen solutions, which can be directly confronted with data of emission Mössbauer spectroscopy. Nevertheless, correlations between the yields of the above mentioned species even under somewhat different conditions are meaningful and indicate on a similarity of the discussed processes in blobs.

\section{Comparison with experiments}

1. Using the data on the yield of $\mathrm{Fe}^{2+}$ (Fig. 1) in frozen aqueous solutions of $\mathrm{NaClO}_{4}, \mathrm{H}_{2} \mathrm{SO}_{4}, \mathrm{HClO}_{4}$, and $\mathrm{HNO}_{3}[6]$ at $77 \mathrm{~K}$ and Eq. (15), $R\left(\mathrm{Fe}^{2+}\right)=$ $\exp \left(-\sum_{i} f_{i} c_{\mathrm{S} i}\right)$, we obtained ratios of $f_{i}$ for different ions, i.e. for electron scavengers existing in these solutions

$$
f\left(\mathrm{H}_{3} \mathrm{O}^{+}\right): f\left(\mathrm{ClO}_{4}^{-}\right): f\left(\mathrm{NO}_{3}^{-}\right) \approx 0.04: 0.05: 0.1 .
$$

These ratios qualitatively agree with the ratios of coefficients $q_{i}$ and $p_{i}$ (see Eq. (13) and Eq. (16)), also characterizing reaction ability of the same ions towards quasi-free electron at room temperature [5, 7]:

$$
\begin{aligned}
& q\left(\mathrm{H}_{3} \mathrm{O}^{+}\right): q\left(\mathrm{ClO}_{4}^{-}\right): q\left(\mathrm{NO}_{3}^{-}\right) \approx(\leq 0.1):(\leq 0.1): 2.4 . \\
& p\left(\mathrm{H}_{3} \mathrm{O}^{+}\right): p\left(\mathrm{ClO}_{4}^{-}\right): p\left(\mathrm{NO}_{3}^{-}\right) \approx(\leq 0.1): 0.1: 3.1 .
\end{aligned}
$$

The proximity of $q$ and $p$ in liquid water at room temperature and their partial deviation from values of $f$, found by means of EMS in frozen media are quite natural. What is important is that all ratios (18)-(20) differ significantly from the ratios

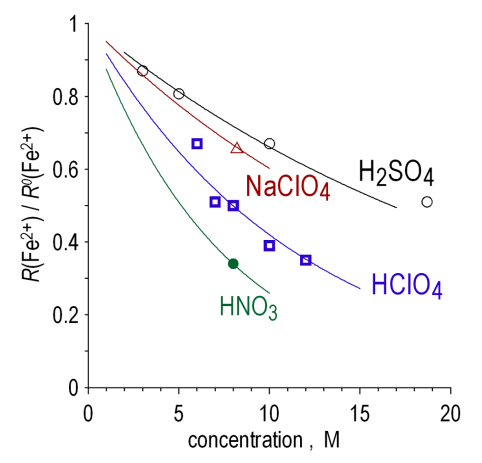

Fig. 1. Relative yields of $\mathrm{Fe}^{2+}$ in acid aqueous solutions frozen at $78 \mathrm{~K}$. Solid lines (exponents) represent Eq. (15) [6]. 
of the reaction rate constants $k\left(\mathrm{e}_{\mathrm{aq}}^{-}+\mathrm{S}_{i}\right)$ between $\mathrm{S}_{i}$ and hydrated electron [5]:

$$
k\left(\mathrm{e}_{\mathrm{aq}}^{-}+\mathrm{H}_{3} \mathrm{O}^{+}\right): k\left(\mathrm{e}_{\mathrm{aq}}^{-}+\mathrm{ClO}_{4}^{-}\right): k\left(\mathrm{e}_{\mathrm{aq}}^{-}+\mathrm{NO}_{3}^{-}\right)=1: 0.1: 1 .
$$

2. Figure 2 demonstrates similar inhibition efficiencies of $G_{\mathrm{H} 2}$ by $\mathrm{Cr}^{2+}$ and $\mathrm{Cu}^{2+}$ ions at room temperature, and similar action of these ions on the $\mathrm{Sn}^{2+}$ yield in frozen $(77 \mathrm{~K})$ aqueous solutions. This fact tells us about similar state and behavior of intrablob electrons in different types of blobs in spite of the large difference in temperature and phase state of the investigated samples.
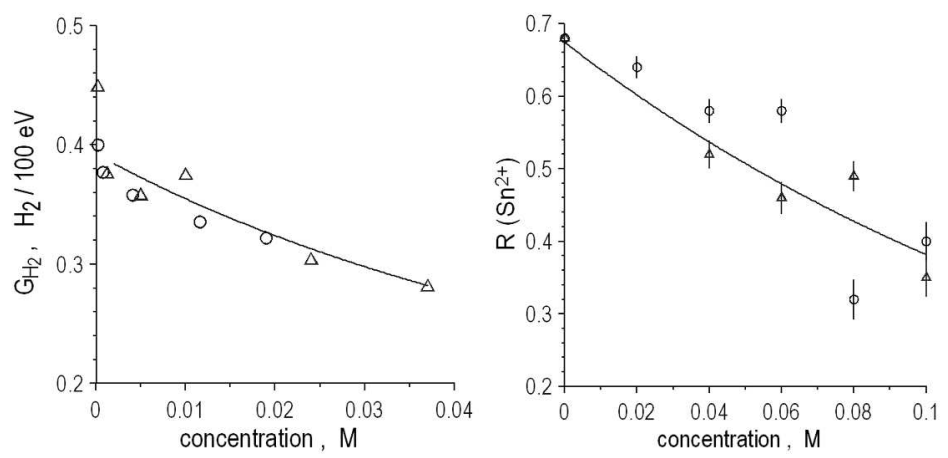

Fig. 2. On the left: dependence of radiolytic hydrogen yields vs. concentration of $\mathrm{Cu}^{2+}(\mathrm{o})$ and $\mathrm{Cr}^{2+}(\Delta)$ ions in aqueous solutions at room temperature [7]. Solid line is an approximation by means of Eq. (14). On the right: dependence of the $\mathrm{Sn}^{2+}$ yield in frozen at $77 \mathrm{~K}$ solutions vs. concentration of salts $\mathrm{CrCl}_{2}(\Delta)$ and $\mathrm{CuCl}_{2}$ (०) [8]. $\mathrm{Sn}^{2+}$ is the final product of ion-electron recombination of the ${ }^{119 \mathrm{~m}} \mathrm{Sn}$ nucleus, after its converted isomer transition. Solid line is calculated with a help of Eq. (16).
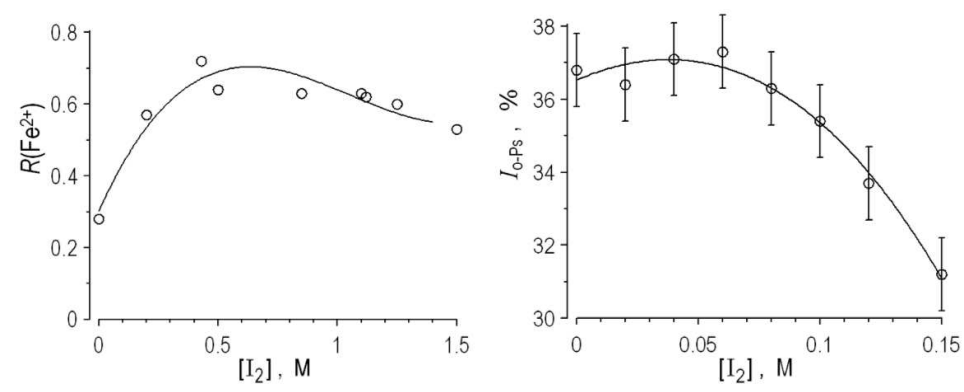

Fig. 3. On the left: $\mathrm{Fe}^{2+}$ yield in frozen at $77 \mathrm{~K}$ ethanol vs. $I_{2}$ concentration [9]. On the right: $o$-Ps yield in liquid cyclohexane at room temperature vs. $I_{2}$ concentration [10]. Solid lines approximate the experimental data.

3. Figure 3 demonstrates complicate behavior of the $\mathrm{Fe}^{2+}$ yield (left) in frozen at $77 \mathrm{~K}$ glassy ethanol vs. concentration of $I_{2}$. In liquid cyclohexane $o-\mathrm{Ps}_{\mathrm{S}}$ formation probability behaves similarly vs. concentration of $I_{2}$ (right). 
4. Figure 4 shows correlation in variation of the yields of $\mathrm{Fe}^{2+}, \mathrm{H}_{2}$, and Ps vs. temperature of the medium. All the yields increase with temperature. Temperature coefficients $\frac{1}{G_{\mathrm{H} 2}} \frac{\mathrm{d} G_{\mathrm{H} 2}}{\mathrm{~d} T}$ and $\frac{1}{I_{o \mathrm{Ps}}} \frac{\mathrm{d} I_{o \mathrm{Ps}}}{\mathrm{d} T}$ are the same, but $\frac{1}{R\left(\mathrm{Fe}^{2+}\right)} \frac{\mathrm{d} R\left(\mathrm{Fe}^{2+}\right)}{\mathrm{d} T}$ is somewhat higher. Probably it is related with variation of the character of solvation of the ions.
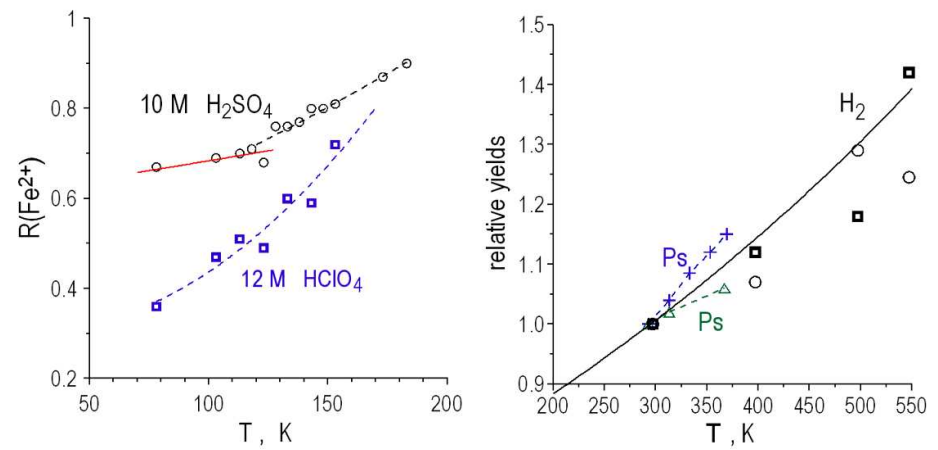

Fig. 4. On the left: temperature dependences of $\mathrm{Fe}^{2+}$ yields in frozen glassy solutions of $10 \mathrm{M} \mathrm{H}_{2} \mathrm{SO}_{4}$ (०) and $12 \mathrm{M} \mathrm{HClO}_{4}$ (ם) [11]. On the right: relative Ps formation probability $(+, \Delta)$ in water and hydrogen yields under the action of fast neutrons $(\square)$ and $\gamma$-rays (o) vs. temperature (see [12] and refs. therein). Solid lines are drawn with the temperature coefficients (see in text).
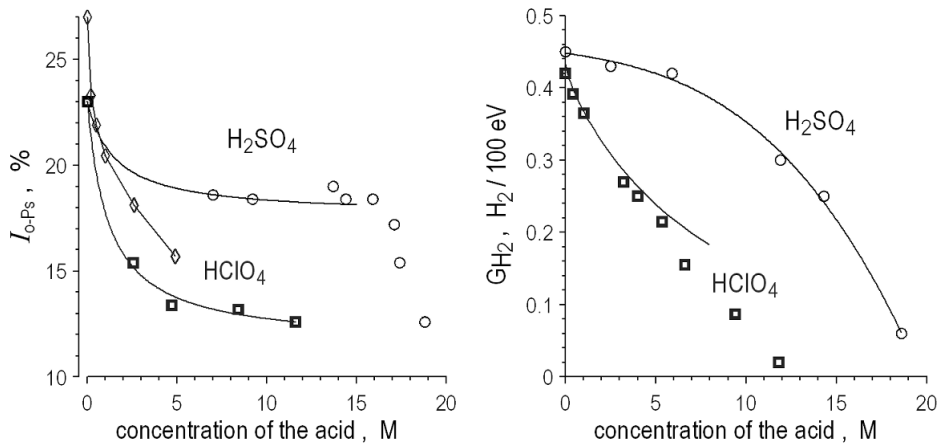

Fig. 5. On the left: $o$-Ps yields in concentrated solutions of $\mathrm{H}_{2} \mathrm{SO}_{4}$ (o - [13]) and $\mathrm{HClO}_{4}(\square-[13] ; \diamond-[14])$. On the right: $\mathrm{H}_{2}$ yields in concentrated solutions of $\mathrm{H}_{2} \mathrm{SO}_{4}(\circ-[15])$ and $\mathrm{HClO}_{4}(\square-[16])$. Solid lines approximate the tendencies in variations of the data.

It is worth mentioning the difference of yields of $\mathrm{Fe}^{2+}$ in solutions of sulphuric and chloric acids, which are clearly seen at low temperatures. This difference holds for the yields of radiolytic hydrogen and $o$-Ps in solution of the same acids at room temperature (Fig. 5). 


\section{Conclusion}

Similar character of an influence of concentration of dissolved substances, temperature and phase state on the yields of the Mössbauer ions ${ }^{57} \mathrm{Fe}^{2+},{ }^{119} \mathrm{Sn}^{2+}$, $o$-Ps, radiolytic $\mathrm{H}_{2}$, and $\mathrm{e}_{\mathrm{S}}^{-}$is revealed. This fact supports our suggestion about similarity of early chemical reactions taking place in positron, electron, and Auger blobs.

\section{References}

[1] Mössbauer Spectroscopy of Frozen Solutions, Eds. A. Vertes, D.L. Nagy, Akademiai Kiado, Budapest 1990.

[2] A.K. Pikaev, Modern Radiation Chemistry. Radiolysis of Gases and Liquids, Nauka, Moscow 1986.

[3] V.M. Byakov, F.G. Nichiporov, Intratrack Chemical Processes, EnergoAtomizdat, Moscow 1985.

[4] V.M. Byakov, J. Phys. IV, Colloque C4, Supplement II 3, 85 (1993).

[5] K.Y. Lam, J.W. Hunt, Radiat. Phys. Chem. 7, 317 (1975).

[6] Yu.D. Perfiliev, L.A. Kulikov, L.T. Bugaenko, A.M. Babeshkin, M.I. Afanasov, J. Inorg. Nucl. Chem. 36, 2115 (1976).

[7] M. Faraggi, Int. J. Radiat. Phys. Chem. 5, 197 (1973).

[8] Yu.D. Perfilev, L.A. Kulikov, V.M. Byakov, S.V. Stepanov, H. Alhatib, L.T. Bugaenko, Khimiya Vysokikh Energii 37, 390 (2003).

[9] Ya.M. Milgrom, Yu.D. Perfilev, A.M. Babeshkin, Khimiya Vysokikh Energii 12, 272 (1978).

[10] B. Levay, P. Hautojarvi, J. Phys. Chem. 76, 1951 (1972).

[11] Yu.D. Perfilev, L.A. Kulikov, A.M. Babeshkin, Zh. Fiz. Khimii LII, 1631 (1978).

[12] V.M. Byakov, S.V. Stepanov, Nukleonika 42, 45 (1997).

[13] S.J. Tao, J.H. Green, J .Phys. Chem. 73, 882 (1969).

[14] V.M. Byakov, V.I. Grafutin, O.V. Koldaeva, E.V. Minaichev, F.G. Nichiporov, Yu.V. Obukhov, O.P. Stepanova, Chem. Phys. 24, 91 (1977).

[15] J.W. Boyle, Radiat. Res. 17, 427 (1962).

[16] D. Katakis, A.O. Allen, J. Phys. Chem. 68, 3107 (1964). 